\title{
Preface to the special issue on "Mathematical modeling and analysis in health and natural sciences"
}

\author{
Bruno Buonomo $^{1}$ D $\cdot$ Lucia Russo $^{2} \cdot$ Nico Stollenwerk $^{3} \cdot$ Constantinos Siettos $^{1}$
}

Received: 29 September 2020 / Accepted: 30 September 2020 / Published online: 7 October 2020

(c) Università degli Studi di Napoli "Federico II" 2020

The quest for developing new mathematical methods for the in-depth modelling and analysis of the behaviour of complex systems with important health, social and economic impact such as in Biology, Medicine and Natural Sciences appears to be a major and timely challenge of our times.

Recent advances in different fields have enhanced and deepened our knowledge in different aspects of disease epidemiology, ranging from the molecular structure of the virus to the impact of the contact transmission network in a population. State-of-the-art mathematical/computational techniques allow the integration of the new information generated on virology, field epidemiology and social behaviour, for example, allowing us to build better and more detailed models in a feedback-based manner.

Developing such mathematical tools to guide public health authorities with projections for the national health systems necessities during an outbreak is urgently needed, as well as long term predictions, while the lockdown measures are gradually lifted, allowing an efficient pandemic response. Indeed, the recent explosion of the COVID-19 pandemic on a planetary level has made it even more urgent: applied mathematicians together with computational engineers, epidemiologists, molecular biologists, virologists join forces in a race against time to combat the pandemic.

$凶 \quad$ Bruno Buonomo

buonomo@unina.it

Lucia Russo

lucia.russo@cnr.it

Nico Stollenwerk

nico.biomath@gmail.com

Constantinos Siettos

constantinos.siettos@unina.it

1 Department of Mathematics and Applications, University of Naples Federico II, via Cintia, 80126 Naples, Italy

2 Institute of Science and Technology for Energy and Sustainable Mobility, Consiglio Nazionale delle Ricerche, Piazzale Tecchio, 80125 Naples, Italy

3 Center for Mathematics, Fundamental Applications and Operations Research, Lisbon University, Campo Grande 1749-016, Portugal 
Thus, the aim of this special issue was to attract high-quality submissions targeting at the development and implementation of new models, as well analytical and numerical analysis methods that have the potential to enhance our understanding in the mechanisms that pertain to the spread and control of newly emergent and re-emergent epidemics. The idea of special issue sprung mainly from the 10th Conference on Dynamical Systems Applied to Biology and Natural Sciences (DSABNS) that took place in Napoli, Italy from 3 to 6 of February 2019.

The articles selected in this special issue of Ricerche di Matematica cover broad topics of mathematical modeling and analysis in health and natural sciences. The authors propose innovative models, analysis and computational methods to study a variety of problems across multiple disciplines including ecology, epidemiology, medicine, population dynamics and finance. Hopefully, the readers will find in this broad overview the inspiration to give their own contribution to future research.

The first article of this Special Issue, authored by Al Basir and Ray, concerns with plant disease control. They focus on mosaic disease, which may cause significant crop losses and food security issues, and propose a model given by nonlinear ordinary differential equations to study the effects on the transmission of the disease of insecticide spraying and removal of infected plants (roguing). The authors also assume that the use of controls depends on the level of awareness about the disease. The best strategies of using spraying, roguing and global advertisement to improve the local awareness are obtained through a suitable optimal control problem.

The second paper fits into the context of population dynamics. Banerjee, Kooi and Venturino present a prey-predator metapopulation model with two patches. In one patch prey and predator interact and the prey is assumed to suffer from the strong Allee effect, i.e. at low levels the prey cannot reproduce and is subject only to mortality, for which its net reproduction rate becomes negative. In the other patch (the safe harbor of the prey), predators cannot migrate and prey grows logistically. Methods of qualitative analysis are used together with theoretical and numerical bifurcation analysis. The authors show that the safe harbor can protect the endangered species under certain parametric restrictions.

In the third paper, by Blyuss and coworkers, the target is again the mosaic disease. In their model the control is performed by using natural microbial biostimulants that, besides improving plant growth, protect plants against infection. The authors show how the use of biostimulants determines whether the mosaic disease is eradicated or is rather maintained at some steady level.

Breda and Liessi propose a pseudospectral collocation method to approximate the eigenvalues of the evolution operators of linear coupled equations, providing rigorous error and convergence analyses as well as numerical tests. This contribution has a strict link with Population Dynamics, due to the increasingly relevant role played in that context by the systems of coupled renewal and retarded functional differential equations.

The next paper, by Buonomo, deals with a compartmental epidemic model called SIRI. The model is implemented by taking into account of the behavioral changes of individuals in response to the available information on the status of the disease in the community. It is shown that the cumulative incidence may be significantly reduced when the information coverage is high enough and/or the information delay 
is short. The specific features of the model are typical of coronaviruses. Therefore, an application is provided by using epidemiological parameter values based on early estimation of the COVID-19 epidemics in China.

The article by Cuomo, Di Somma and Sica focuses on comparing two numerical methods when applied to the Heston model in finance. The first method is the alternating direction implicit (ADI) method, which is quite commonly used in finance but suffers a problem of high computational cost in high-dimensional problems. The second method is the radial basis functions (RBF) method. Among the results obtained, the authors show that RBF seems to be more precise than ADI by inputting the same grid and market data.

In their contribution, Grundland and Hariton show how the approximate symmetry analysis of a nonlinear wave equation with small dissipation combined with singularity analysis provides exact analytic solutions. The analysis is performed by using the Lie symmetry algebra of the wave equation. The conjugacy classes of the one-dimensional subalgebras of this Lie algebra are identified. It is shown that the subalgebra classification of the integro-differential form of the nonlinear wave equation is much larger than the one obtained from the original wave equation. A systematic use of the symmetry reduction method allows the authors to find new invariant solutions of the wave equation.

The problem of damaging pest in agriculture is the focus of the paper by Kumar Dhar and Bhatti, where a model given by three differential equations including two delays is studied. The model describes the interaction between plant, pest and natural enemy. The two delays take into account of gestation delays for pests and natural enemies, respectively. A detailed analysis is performed and it is shown, in particular, that delay-induced oscillating behavior through Hopf bifurcation may occur.

Marasco, Giannino and Iuorio introduce a spatial model to study the effect on the coexistence of two competing plant species of both inter-specific and intra-specific plant-soil feedback induced by toxicity. By performing both qualitative analysis and computational methods, the authors show the existence of a rich spectrum of ecological scenarios, such as competitive exclusion, stable coexistence, and spatio-temporal patterns. In particular, their results reveal that inter-specific plant-soil feedback can lead to stable coexistence of the two plant species, whereas the absence of such feedback would imply competitive exclusion. Therefore, the authors conclude that inter-specific plant-soil feedback provides a facilitation mechanism and enlarges the range of species coexistence and then biodiversity.

An application of dynamical systems to medicine is given by Parajdi who considers a system modeling cell dynamics after bone marrow transplantation in chronic myeloid leukemia. A detailed analysis of the stability of the steady-states is provided together with numerical simulations. Both the cases of chronic and accelerated-acute phases of the disease are considered.

Rabiu, Willie and Parumasur develop an epidemic model designed for infectious diseases like influenza virus and severe acute respiratory syndrome (SARS). The feature of the model is that the infectious are divided in asymptomatic and symptomatic ones. Quarantine and vaccine are considered as mitigation strategies. It is shown that the phenomenon of backward bifurcation may take place, where a stable disease-free equilibrium co-exists with a stable endemic equilibrium. This phenomenon is elim- 
inated either when the vaccine is perfect (that is, it guarantees $100 \%$ efficacy and potency) or when a mass-action incidence is adopted.

The paper by Raïssi, Serhani and Venturino focuses on an optimal control in biological wastewater treatment. The authors propose a non linear mathematical model describing the process of biodegradation of organic pollutants. A qualitative analysis is provided to assess the steady states of the model together with their stability properties. The sensitivity of the persistence equilibrium is also investigated in terms of the model parameters. Then, an optimal control problem is set with the aim of keeping pollutant and fungi effluents close to zero and permitting a continuous activity of the plant by feeding pollutant.

Qualitative analysis and optimal control are also the main tools used by Raw, Tiwari and Mishra to study a three species plankton-fish system incorporating external toxicity and harvesting. The authors show that oscillation and chaos may occur but the harvesting effort has a stabilizing effect. Furthermore, an optimal control problem is set to maximize the benefit as well as the conservation of the ecosystem.

The last but not least paper of this Special Issue, authored by Wang and coauthors, deals with an in-host virus model with diffusion and distributed delays. The global dynamics of the infection equilibrium is investigated by constructing appropriate Lyapunov functionals. In this way, the authors obtain sufficient conditions for the persistence of the virus patient and, therefore, for the infection to become chronic.

The guest editors take the last lines to fully acknowledge the editor-in-chief, Prof. S. Rionero, for having provided us with the opportunity to publish this special issue. They would also like to thank all the authors for their contributions and the reviewers for their work.

\section{Compliance with ethical standards}

Conflict of interest On behalf of all authors, the corresponding author states that there is no conflict of interest.

Publisher's Note Springer Nature remains neutral with regard to jurisdictional claims in published maps and institutional affiliations. 